\title{
Aplicación de los modelos de elección discreta para priorizar y seleccionar usuarios de salas de cirugía
}

Application of Discrete choice models for prioritize and select users of operating rooms

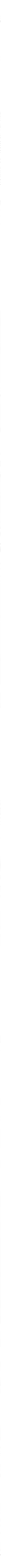




\title{
Aplicación de los modelos de elección discreta para priorizar y seleccionar usuarios de salas de cirugía ${ }^{1}$
}

\section{Application of Discrete choice models for prioritize and select users of operating rooms}

\section{Juan Fernando Guarín Castro², Carlos Eduardo Díaz Bohórquez ${ }^{3}$}

Universidad Industrial de Santander UIS, Bucaramanga, Colombia

Artículo recibido en abril de 2016; aceptado en junio de 2016

Citación del artículo: Guarín, J. \& Díaz, C. (2016). Aplicación de los modelos de elección discreta para priorizar y seleccionar usuarios de salas de cirugía. I+D Revista de Investigaciones, 8(2). pp 54 - 62

\begin{abstract}
Resumen
Este artículo explora una metodología cuantitativa basada en una distribución de probabilidad Logit para establecer las prioridades en la selección de pacientes acumulados en las listas de espera de los servicios de cirugía en las instituciones prestadoras de servicios de salud. Se evaluó un conjunto de factores asociados a los resultados de las intervenciones quirúrgicas como efecto de la interacción entre la patología del paciente y las fortalezas operativas de la IPS, para estimar el impacto en la mejora de la calidad de vida del paciente a través de un índice en escala de intervalo. Esta forma de priorizar supera las limitaciones de los resultados de los instrumentos tradicionales para la medición de la variable, por medio de un modelo de selección más eficiente.
\end{abstract}

Palabras clave: priorización de pacientes, variables latentes, modelos de elección discreta, función Logit, modelo de Rasch.

\begin{abstract}
This paper explores a quantitative methodology based on a probability distribution Logit to establish priorities in the selection of patients accumulated waiting in lists for surgery services in the Health services institutions. A set of associated factors at the surgical intervention results was evaluated, such effect of the interaction between the patient's pathology and the IPS operative skills, for to estimate the achieved impact in the improve of the patient's quality life through an index in interval scale. This way of prioritizing overcomes the limitations of the results of traditional instruments for measuring the variable, through a more efficient selection model.
\end{abstract}

1. Artículo científico de enfoque cuantitativo, componente parcial de un proyecto de investigación en curso perteneciente al área de Optimización multiobjetivo, subárea de Metaheurísticas, desarrollado en el Grupo de Investigación Opalo de la Universidad Industrial de Santander (Colombia), institución que financia el proyecto. Dirección: Carrera 27 Calle 9 Ciudad Universitaria. Fecha de inicio: agosto de 2014, fecha de terminación: en

curso

2. Ingeniero Industrial, Universidad Pontificia Bolivariana, UPB, Seccional Bucaramanga. Candidato a MSc. Ingeniería Industrial, Universidad Industrial de Santander, UIS. Docente Universitaria de Investigación y Desarrollo UDI (Bucaramanga, Colombia). Investigador del Grupo de Investigación Opalo, Universidad Industrial de Santander, UIS (Colombia). Dirección: Carrera 27 Calle 9 Ciudad Universitaria. PBX: + 5716344000. jugua7612@hotmail.com

3. Ingeniero Industrial, Universidad Industrial de Santander. MSc. Ingeniería Industrial, Universidad de los Andes. Docente UIS, director Grupo de Investigación Opalo, Universidad Industrial de Santander, UIS (Colombia). Dirección: Carrera 27 Calle 9 Ciudad Universitaria. PBX: + 5716344000. cediazbo@uis.edu.co 
Key words: Patient prioritization, latent variable models, discret choice models, Logit function, Rasch model.

Partiendo de la premisa de que el objetivo principal de las empresas es la obtención de beneficios económicos que garanticen su viabilidad y sostenibilidad en el tiempo (Sallenave, 1994), se han diseñado múltiples herramientas que sirven como instrumento para medir de forma eficiente este logro.

En parte, el hecho se facilita porque se puede demostrar, gracias a la aritmética, por ejemplo, que si una empresa obtuvo en los dos últimos años unos beneficios de 2 y 4 millones, entonces las utilidades del segundo año respecto del primero son del doble, y si los beneficios hubiesen sido de 2 y 6 millones, entonces serían del triple respectivamente. Esto ocurre porque la variable de análisis (el beneficio) es una variable observable de tipo cuantitativo, que está expresada en escala de razón.

Sin embargo, cuando se quieren medir variables no observables como "la calidad", el "nivel de satisfacción" o el "estado de salud y bienestar" de una población, los resultados generalmente están expresados por medio de puntajes (Pride, 2015) obtenidos con instrumentos que tienden a presentar valoraciones no del todo claras: ¿acaso se podría afirmar que alguien que ha calificado con 4 su nivel de satisfacción por el servicio recibido en un hotel se habrá sentido exactamente la mitad de bien que alguien que calificara su experiencia con un 8 ?

En este caso, la variable es de tipo cualitativo y está expresada en una escala ordinal (Anderson, 2009).

Habida cuenta de lo anterior, en la actualidad uno de los principales retos a los que se enfrentan los tomadores de decisiones para balancear sus resultados empresariales consiste en integrar en un solo escenario varios objetivos generalmente en conflicto (p. ej. económicos y sociales) que a su vez tienen diferentes tipos de escala de medida (Taha, 2008).

En el sector salud este balance suele ser de nivel crítico, pues el desarrollo de las operaciones que llevan a cabo las instituciones prestadoras de servicios de salud (IPS) se ha tenido que estructurar entre el resultado que se puede lograr para el beneficio del usuario en el cuidado de su salud, y lo que el sistema de salud puede gestionar manteniendo un margen de rentabilidad favorable (Alemi \& Gustafson, 2010).

En efecto, la atención en salud se convierte en una actividad viable cuando las IPS mantienen su nivel de solvencia y los usuarios obtienen un nivel satisfactorio de calidad en la prestación de un servicio que aumenta su calidad de vida.

Teniendo en cuenta la importancia que tiene el área de cirugía dentro del grupo integral de servicios de salud (Cardoen, 2010), es necesario un mecanismo que permita establecer de forma precisa cuáles son los procedimientos registrados en la lista de espera que maximizan el impacto positivo en la calidad de vida de los pacientes, de manera que se garantice la selección de aquellos con el mayor nivel de calidad posible en la atención.

Se busca con esta selección que en un análisis posterior, por ejemplo, se pueda sopesar el resultado obtenido contra los datos que contienen información sobre los procedimientos que maximizan el impacto económico, haciendo lo propio para encontrar el punto que garantice un equilibrio.

Se propone diseñar entonces una herramienta que permita medir la calidad de la atención derivada de la gestión en el establecimiento de la prioridad (Apella, 2009). Aunque en algunos trabajos realizados por autores como Derret \& Devlin (2003); Ortún, Pinto \& Puig-Junoy (2001); Oudhoff \& Rimmermans (2007) y Sabik \& Lie (2008), se han ensayado modelos matemáticos para estimar priorizaciones, no se ha evidenciado en la literatura un modelo que utilice las distribuciones de probabilidad para establecer resultados en términos de la interacción que se da entre el paciente, su patología y la capacidad de tratamiento que pueda brindarle la IPS que atiende su caso.

Este artículo presenta una metodología (Kerlinger \& Lee, 2002) para medir de forma válida y confiable el valor de la variable no observable "Nivel de calidad asistencial del paciente (NCAP)" que cada usuario posee, independiente de que se encuentre en determinada lista de espera, por medio de una distribución de probabilidad que describe el impacto que tienen los resultados de las intervenciones quirúrgicas en la mejora de la calidad de vida del paciente.

\section{Método}

El objetivo de este trabajo es formular, por medio de una función matemática, un modelo para resolver el problema de priorización de pacientes, estableciendo una variable no observable (Montgomery, 1995) denominada "Nivel de calidad asistencial (NCAP)". Dicha variable es el eje de referencia para el diseño de la escala donde los diferentes usuarios van ser medidos con base en información proveniente de los juicios y valoraciones que tradicionalmente los especialistas hacen de sus patolo- 
gías, por medio de métodos cualitativos. La información de la variable se convirtió de cualitativa a cuantitativa en escala de intervalo, utilizando las respuestas a un instrumento de medición fundamentado en los modelos de elección discreta binaria, aplicando las propiedades de una de sus distribuciones de probabilidad, llamada función Logit.

El perfil del estudio es de carácter teórico, con un diseño correlacional de corte transversal y respondió la siguiente pregunta de investigación: ¿Cuáles son esas cirugías que están en la lista de espera, que al seleccionarlas como grupo en una iteración para el uso de una sala de cirugía, producen el mayor nivel de calidad asistencial?

\section{Participantes}

El resultado de la estructura propuesta es un modelo teórico, razón por la cual sus creadores -y además autores del presente artículo- son los participantes del proyecto. No obstante, los datos de las instancias de prueba se obtuvieron gracias a la disposición y colaboración de la Dirección Administrativa y Financiera del Instituto del Corazón de Bucaramanga IPS.

\section{Materiales e instrumentos}

\section{Instrumentos de recolección de datos:}

- Cuestionario para la valoración de criterios de prioridad para los eventos en salas de cirugía (Briones, 2008). Por medio de este instrumento se evaluaron grupos de variables asociados al tiempo de espera (Asua \& Taboada, 2005), desempeño de la recuperación según el diagnóstico (Sánchez \& Abellán, 2008), impacto en la calidad de vida (Hauch \& Smith, 2004), y nivel de éxito del procedimiento, caracterizaciones por edad, por género y por patologías conjuntas (Espallargues, Gallo \& Sampietro, 2000).

- Cuestionario de factores asociados al nivel de prestación del servicio. Por medio de este instrumento (Edwards, 1997) se recopiló información sobre el nivel de las habilidades, conocimientos y experiencia de la institución (HCE) (Ministerio de Salud, 2015) respecto de las patologías tratadas, así como de las políticas para los convenios y modelos de contratación con las EPS (Ministerio de la Protección Social, 2008).

\section{Bases de datos}

- Instancias de prueba, llevadas a cabo con bases de datos creadas mediante simulaciones de archivos digitales de listas oficiales de espera con sus respectivos usuarios de quirófanos.

\section{Programas}
- WINSTEPS (Linacre)
- SPSS (Corp. IBM, 2016)

\section{Procedimiento}

El nivel de calidad asistencial del procedimiento (NCAP) se configuró como una variable no observable utilizada para describir el grado de importancia que tiene un procedimiento en el logro de un nivel aceptable de la calidad de vida del paciente, grado que se determina por medio de la presencia o ausencia de ciertos rasgos (Hair \& Tatham, 2001) que solo pueden ponerse en evidencia y ser detectados por medio de un conjunto de ítems que son conocidos como reactivos (enunciados en forma de pregunta diseñados para recolectar evidencia de la presencia o ausencia de una característica o rasgo en un objeto evaluado) (Cohen, 1979).

La medida de la variable se definió por el resultado de la interacción entre el contexto del usuario y la patología para la que requiere un procedimiento, y el contexto del procedimiento y la experiencia de tratamiento en la IPS.

El contexto del procedimiento y la experiencia de tratamiento en la IPS fueron estructurados por medio de un conjunto de ítems, definidos y diseñados específicamente para la detección de aquellos rasgos determinantes que dan cuenta del nivel de calidad asistencial. Estos ítems, al construirse y ordenarse estratégicamente, pueden agruparse en componentes o factores (De la Garza, Morales \& González, 2013).

El nivel que presentan los usuarios en cada uno de los rasgos se asoció al resultado de sus respectivos comportamientos frente al conjunto de ítems.

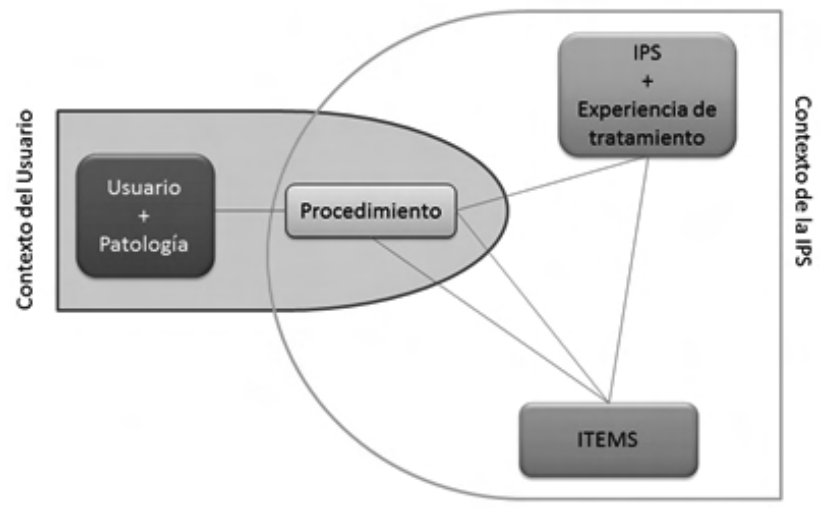

Figura 1.

Esquema de interacción entre los contextos del usuario y de la IPS 
Como cualquier otra variable continua, el "nivel de calidad asistencial del procedimiento" se representó por medio de una línea recta a lo largo de la cual se situaron los ítems y los usuarios de las salas de cirugía. A medida que se avanza hacia la derecha de la línea, cada punto implica "mayor cantidad" de la variable.

¿Cuál fue el criterio para ubicar los ítems y los usuarios en la línea de medida?

En la Figura 2 se observa el modo en que usuarios e ítems se pueden encontrar distribuidos a lo largo de la línea que representa la variable no observable, que va de cero a infinito.

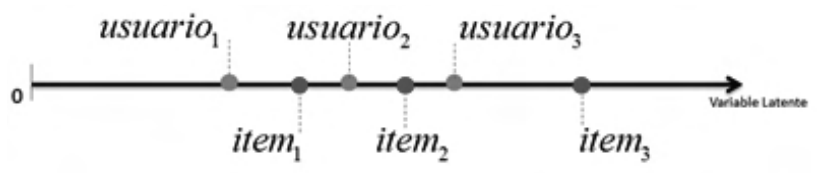

Figura 2.

\section{Representación ítems y usuarios sobre la línea de la variable no observable}

Si el rasgo que representa el ítem 1 es el de menor medida, este estará presente en los usuarios 2 y 3 , pero no en el usuario 1. También se puede observar que el rasgo que representa el ítem 3 no está presente en ninguno de los usuarios (Thorndike, 1989).

De este modo, se pudo concluir que un usuario tendrá un mayor nivel de calidad asistencial en la medida en que presente mayor número de rasgos.

Habida cuenta de lo anterior, para que se pudiera afirmar que un usuario $U$ posee un determinado rasgo caracterizado por el ítem I fue necesario que este rasgo se detectase mediante una evidencia, que se pone de manifiesto cuando para las medidas de $U$, I se cumpla la siguiente condición: U > I.

Al existir varios usuarios con diferentes niveles de calidad asistencial, sus diferencias se hicieron manifiestas a través del conjunto de rasgos que componen los ítems, y permitieron establecer sus posiciones respecto a ellos. La evidencia requerida para establecer la ausencia o presencia del rasgo en los usuarios se pudo determinar por medio de un modelo dicotómico de elección discreta de respuesta para el conjunto de los ítems, en el cual, si la respuesta del usuario $U$ al ítem I tomó el valor de $X=1$ se afirmó que el usuario $U$ posee el atributo caracterizado por el ítem I, y si el valor fue $X=0$, se consideró lo contrario.

Una matriz que recogiera la información de las respues- tas a los ítems, ordenada de menor a mayor respecto a la valoración del rasgo latente que caracteriza cada uno de los ítems, y a su vez ordenara a los usuarios respecto de aquel que tiene el menor puntaje hasta aquel de mayor puntaje, tendría una estructura ideal similar a la de la Tabla 1.

Tabla 1

Modelo de distribución ideal de respuestas

\begin{tabular}{|c|c|c|c|c|c|c|c|}
\hline & Item $_{(1)} \mid$ & tem $_{(2)} I$ & tem $_{(3)} I$ & $\operatorname{tem}_{(4)} 1$ & tem $_{(5)} I$ & $\operatorname{tem}_{(6)}$ & $\begin{array}{l}\text { Puntajes de } \\
\text { los Usuarios }\end{array}$ \\
\hline Usuario $_{(1)}$ & 1 & 0 & 0 & 0 & 0 & 0 & 1 \\
\hline Usuario $_{(2)}$ & 1 & 1 & 0 & 0 & 0 & 0 & 2 \\
\hline Usuario $_{(3)}$ & 1 & 1 & 1 & 0 & 0 & 0 & 3 \\
\hline Usuario(4) $_{(4)}$ & 1 & 1 & 1 & 1 & 0 & 0 & 4 \\
\hline Usuario(5) $_{(5)}$ & 1 & 1 & 1 & 1 & 1 & 0 & 5 \\
\hline Usuario $_{(6)}$ & 1 & 1 & 1 & 1 & 1 & 1 & 6 \\
\hline $\begin{array}{l}\text { Puntajes de } \\
\text { los Ítems }\end{array}$ & 6 & 5 & 4 & 3 & 2 & 1 & \\
\hline
\end{tabular}

La matriz de los datos recopilados a través de los instrumentos se comparó con el comportamiento de los datos de un modelo de elección discreta como referente, con el objetivo de determinar la posibilidad de que estos presentaran un patrón de ajuste, y así poder usar su función de distribución de probabilidad.

Este hecho permitió formular un modelo en el cual un usuario $U$ que tiene una determinada patología $\mathrm{H}$, al interactuar sobre el ítem I que caracteriza aspectos de la IPS sobre el procedimiento $T$ produjera una respuesta $X$. ¿Todos los unos significan lo mismo? En los modelos de elección discreta esa respuesta, bien sea para 1 o para 0 , surge de la relación de interacción que se produjo entre la patología del usuario y la experiencia de la IPS en el manejo del procedimiento. Para poder expresarla se requirió de la búsqueda de un método que produjera una respuesta que siguiera el modelo de la Tabla 1. Los modelos de elección discreta del tipo Logit cumplen con estos criterios (Kenneth, 2009).

Si en un juego de azar hubiese una regla o condición que asignara, por ejemplo, el valor de "1" al hecho de haber sacado un seis, y un cero al hecho de sacar cualquier otro número, ese " 1 " tiene que ver con las probabilidades: no es lo mismo sacar un seis al tirar un dado, que sacarlo de una bolsa donde hay balotas numeradas del 1 al 50 . Sin embargo, para cualquiera de los dos escenarios si se obtiene un seis, aparecerá un "1".

De la misma forma en que se puede estimar lo difícil que puede ser sacar un " 1 " en el ejemplo anterior, se pudo estimar la probabilidad de ese " 1 " $y$ de ese " 0 " que aparecen en la tabla de los datos (Liao, 1994).

Al haber encontrado un modelo probabilístico para la estimación de la respuesta al cual se ajustan los datos, se 
logró convertir una escala ordinal en una escala numérica continua (Wright, 1977).

El modelo se describió por medio del comportamiento de dos parámetros:

$\beta_{(n)}=$ la complejidad de la patología del usuario $U_{(n)}$

$\delta_{(i)}=$ la habilidad de la IPS en el manejo del procedimiento en el criterio I

Los anteriores parámetros se pudieron relacionar para calcular la probabilidad de acierto (obtener el valor de 1 para la variable $X)$ a un ítem mediante el diseño de una función en términos de la diferencia $(\beta-\delta)$ a través de un intervalo de 0 a 1 por medio de la siguiente transformación Logit:

$$
P\left\langle X_{i}=1 \mid \beta_{n}-\delta_{i}\right\rangle=\frac{e^{\left(\beta_{n}-\delta_{i}\right)}}{1+e^{\left(\beta_{n}-\delta_{i}\right)}}
$$

Ecuación 1. (Rasch, 1981).

Utilizando la ecuación 1 para encontrar el valor de la probabilidad de obtener $\mathrm{X}=1$ en función de los parámetros ( , ) se operó con la siguiente lógica: si la diferencia entre los parámetros es muy grande sea cual sea, y además positiva, entonces la probabilidad de obtener el acierto en el ítem $(=1)$ tiende a 0 , lo que se interpretó como un hecho en el que la complejidad de la patología del usuario fue tan grande y la experiencia de la IPS tan poca, que es casi imposible que el rasgo de calidad asistencial que define ese criterio se encontrara en el usuario.

Por el contrario, si la diferencia es muy grande, y negativa, la probabilidad tiende a 1 , lo que se interpreta como el hecho de que la IPS tuviera una experiencia y habilidad tan grande, y la complejidad del procedimiento fuera $\tan$ pequeña, que sería muy probable que el usuario tuviera el rasgo de calidad asistencial que define ese criterio y su respuesta fuese $(=1)$ con una probabilidad muy alta. Para el caso en que la diferencia tendió a cero, se estimó un $50 \%$ de probabilidad de que el usuario tuviera el rasgo que caracteriza al ítem y por tanto, sería tan probable que obtuviese un " 1 " como un " 0 ".

El problema de hallar el valor de los parámetros para establecer los montos de las diferencias asociadas a su nivel de probabilidad se resolvió estimando uno de ellos por medio de los datos recopilados en la matriz de los datos que contiene la información.

En este caso, al transformar la probabilidad de acierto de los ítems en una función se utilizaron los datos de las res- puestas a los ítems para reemplazar el parámetro correspondiente al nivel de habilidad y experiencia de la IPS, como se expresa en por medio de la siguiente ecuación:

$$
(\beta-\delta)=\ln \left(\frac{p}{(1-p)}\right)
$$

Ecuación 2. (Tristán, 2001).

El cálculo de $\mathrm{p}$ no presentó mayor dificultad, debido a que corresponde al valor de las proporciones de las respuestas a los ítems en la matriz de datos (ver Figura 3).

\begin{tabular}{ccccccc}
\hline $\begin{array}{c}\text { Puntajes de } \\
\text { los items }\end{array}$ & 6 & 5 & 4 & 3 & 2 & 1 \\
\hline $\begin{array}{c}\text { Total } \\
\text { Usuarios }\end{array}$ & 6 & 6 & 6 & 6 & 6 & 6 \\
$\begin{array}{c}\text { Aciertos } \\
\boldsymbol{p}\end{array}$ & 6 & 5 & 4 & 3 & 2 & 1 \\
\hline
\end{tabular}

Figura 3.

Calculo de p a través de los datos obtenidos en la Tabla 1

De manera muy conveniente, el comportamiento de la función con la que se trataron las respuestas se ajustó perfectamente al modelo de probabilidad, como se puede apreciar en la Tabla 2 y en la Figura 4.

Tabla 2

Comportamiento de la función In (p/1-p)

\begin{tabular}{cccc}
\hline $\mathbf{p}$ & $\mathbf{1 - p}$ & $\mathbf{P} /(\mathbf{1}-\mathbf{p})$ & $\mathbf{L n}(\mathbf{p} /(\mathbf{1}-\mathbf{p})$ \\
\hline 1 & 0.0 & - & - \\
0.99 & 0,01 & 99,00 & 4,60 \\
0.90 & 0,1 & 9,00 & 2,20 \\
0.80 & 0,2 & 4,00 & 1,39 \\
0.7 & 0,3 & 2,33 & 0,85 \\
0.5 & 0,5 & 1,00 & 0,00 \\
0.4 & 0,6 & 0,67 & $-0,41$ \\
0.3 & 0,7 & 0,43 & $-0,85$ \\
0.1 & 0,9 & 0,11 & $-2,20$ \\
0.01 & 0,99 & 0,01 & $-4,60$ \\
0 & 1 & 0 & - \\
\hline
\end{tabular}

Los procedimientos para la validación de muestras se fundamentan en los modelos de Máxima verosimilitud (Habermann, 1977), sin embargo, existen alternativas menos complejas como el algoritmo PROX, desarrollado por Leslie Cohen (1979), con el cual se obtuvieron resultados equivalentes (Wright \& Douglas, 1976; Wright \& Mead, 1975).

El proceso se repitió a lo largo de una serie de iteraciones seleccionando y etiquetando un grupo de usuarios -quienes permanecieron siempre presentes en el grupo 


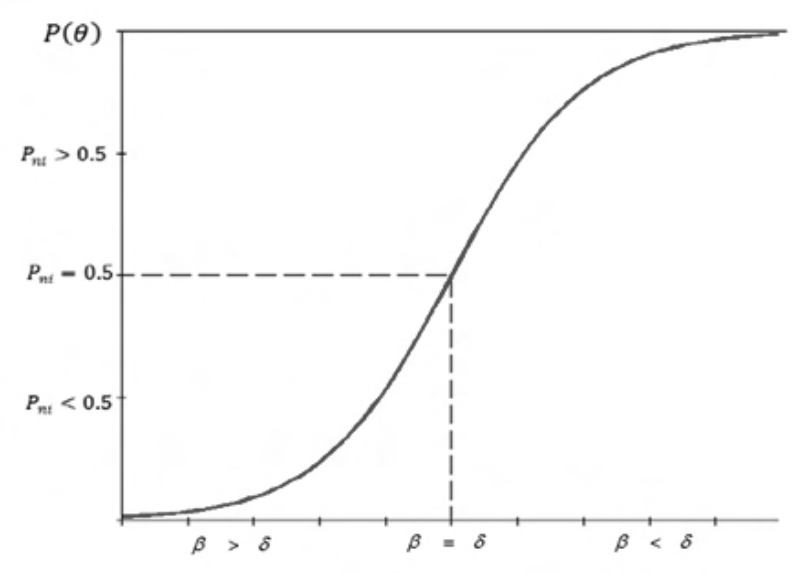

Figura 4.

Comportamiento de la función $\ln (p / 1-p)$

de población- para someterlos a un proceso de trazabilidad de resultados. Asimismo, se extrajeron e introdujeron nuevos miembros a la población reemplazando a aquellos que no estaban etiquetados.

\section{Resultados}

Mediante los modelos de elección discreta se logró evaluar la posibilidad de que un usuario fuera incluido en la K-iésima programación de salas de cirugía, al establecer un método para medir de forma válida y confiable su nivel de prioridad para hacer uso de las salas.

Se pudo variar la configuración de la población de usuarios (extraer e introducir nuevos usuarios) sin que se afectara la medida individual de los usuarios que se evaluaron.

En la Figura 5 se puede observar la línea de la variable en forma vertical (punteada) donde se ubicaron los usuarios y los ítems al realizarse la respectiva iteración del programa.

En la gráfica de la izquierda se muestra uno de los resultados de las primeras pruebas, en donde los ítems representados en el mapa de preguntas no se distribuyeron uniformemente a lo largo de la variable, lo que obligó a ajustar el cuestionario de ítems, el cual, una vez ajustado en varias oportunidades, presentó finalmente el comportamiento que se observa en la gráfica de la derecha.

En la Figura 6 se presenta un segmento de la tabla de resultados y se pueden observar en la columna "Measure" los datos que se calcularon, ya transformados en escala de intervalo para los primeros usuarios. Esta tabla fue generada con el programa Winsteps (Bond \& Fox, 2001).
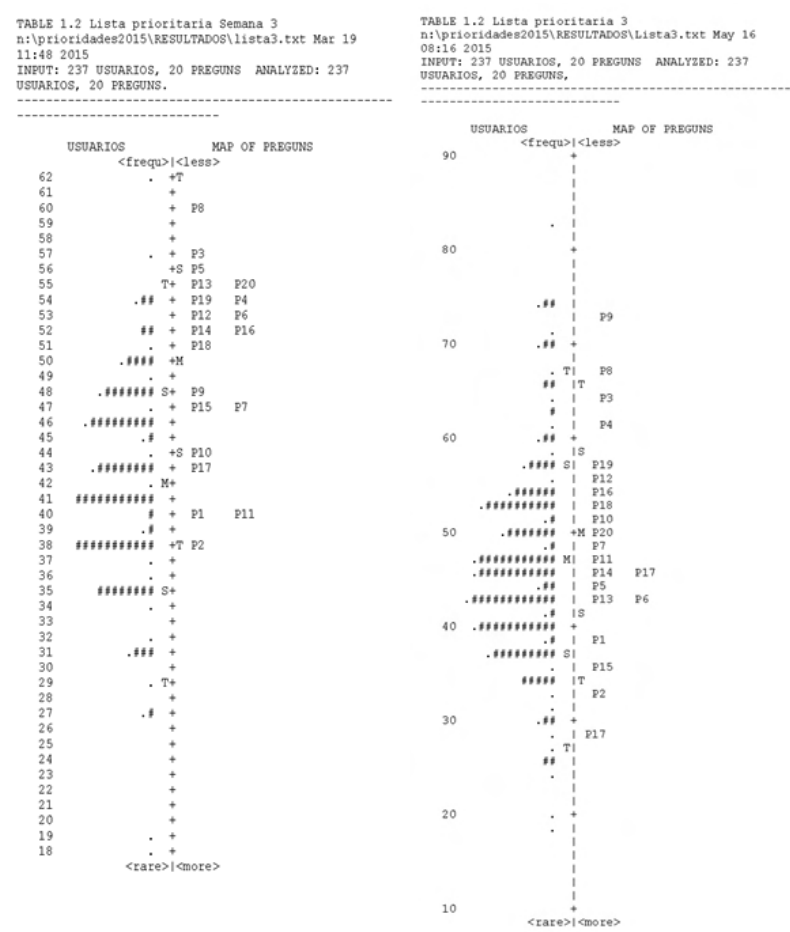

Figura 5.

Línea continua vertical donde se ubican el usuario, y el cuestionario antes y después de ajustes

\begin{tabular}{|c|c|c|c|c|c|c|c|c|}
\hline \multirow[b]{2}{*}{$\begin{array}{l}\text { I ENTRY } \\
\text { I NUMBER }\end{array}$} & \multicolumn{7}{|c|}{ USUARIO STATISTICS: MEASURE ORDER } & \multirow[b]{2}{*}{ ALUneNo } \\
\hline & $\begin{array}{l}\text { RAW } \\
\text { SCORE }\end{array}$ & COUNT & MEASURE & ERRORIMass & $\begin{array}{l}\text { FIT I OUT } \\
\text { ZSTDIMNSQ }\end{array}$ & $\operatorname{FiT}_{2 S T}$ & $\begin{array}{l}\mathrm{RE} \\
\mathrm{R}, \mathrm{I}\end{array}$ & \\
\hline & & & & & & & & 1 \\
\hline 112 & $\begin{array}{l}19 \\
18\end{array}$ & & & & $2_{2}^{3}$ & & .181 & \\
\hline $\begin{array}{l}112 \\
294\end{array}$ & 1 & & & 1 & $\begin{array}{r}.21 .92 \\
-.71 . .32\end{array}$ & -1. & $\begin{array}{l}.1611 \\
.661\end{array}$ & \\
\hline 297 & 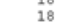 & & & & -.71 .32 & -1.11 & .661 & \\
\hline 303 & 1 & & & 7.69991 & -.71 .32 & -1.11 & .661 & \\
\hline 306 & 18 & & 8 & 7.69991 .67 & $\begin{array}{lll}-.71 & .32\end{array}$ & -1.11 & .661 & \\
\hline 308 & 18 & & & 7.69991 .67 & & & .661 & \\
\hline 311 & 18 & & & & & & .661 & \\
\hline 312 & & & & 7. & - & & .661 & \\
\hline $\begin{array}{l}318 \\
319\end{array}$ & 1 & & & 7.6 & -.71 .32 & -1 & .661 & \\
\hline $\begin{array}{l}319 \\
321\end{array}$ & 1 & & & 7.69991 .67 & $-.7 \mid .32$ & -1.11 & .661 & \\
\hline $\begin{array}{l}321 \\
322\end{array}$ & 1 & & $\begin{array}{l}74.4058 \\
74.4058\end{array}$ & $\begin{array}{r}7.69991 .67 \\
7\end{array}$ & $-.71 \cdot .32$ & -1.11 & .661 & 1 \\
\hline $\begin{array}{l}322 \\
326\end{array}$ & $\begin{array}{l}18 \\
18\end{array}$ & & $\begin{array}{l}74.4058 \\
74.4058\end{array}$ & $\begin{array}{r}7.69991 .67 \\
7.69991 .67\end{array}$ & $\begin{array}{l}-.71 .32 \\
-.71 .32\end{array}$ & $\begin{array}{l}-1.11 \\
-1.11\end{array}$ & $\begin{array}{l}.6661 \\
.661\end{array}$ & \\
\hline 328 & 1 & & 74.4 & 7.69991 .67 & -.71 .32 & -1.11 & .661 & \\
\hline 724 & & & & 7. & -.41 & & .511 & \\
\hline 310 & & & & 7 . & -.71 & & 66 & \\
\hline 327 & 17 & & & 11.67 & -.71 & & .661 & \\
\hline $\begin{array}{l}300 \\
304\end{array}$ & & & & 21.66 & -.71 & -1.11 & .671 & \\
\hline $\begin{array}{l}304 \\
309\end{array}$ & 1 & & & $\begin{array}{l}7.74921 .66 \\
7.7492\end{array}$ & $\begin{array}{ll}-.71 & .32 \\
-.71 & .32\end{array}$ & -1.11 & $\begin{array}{l}.671 \\
671\end{array}$ & \\
\hline & & & & 7.92301 .96 & $\begin{array}{l}-.71 . .32 \\
-.111^{3}\end{array}$ & -1.11 & $\begin{array}{l}.641 \\
.241\end{array}$ & \\
\hline 10 & & & & .91 & -.21 & -.51 & .401 & \\
\hline 10 & & & & & & 1 & & \\
\hline 111 & 1 & & & & & & & \\
\hline 125 & 1 & & & & -.31 & -.5 & .431 & \\
\hline $\begin{array}{l}223 \\
293\end{array}$ & 1 & & & 11.83 & $-.41 \quad .58$ & -.81 & .511 & \\
\hline $\begin{array}{l}293 \\
299\end{array}$ & & & & $\begin{array}{l}11.77 \\
11 \\
1.85\end{array}$ & $-.6 \mid .66$ & -.61 & .531 & \\
\hline 30 & & & & $\begin{array}{l}.85 \\
.72\end{array}$ & $\begin{array}{l}-.412 .08 \\
-.71 .50\end{array}$ & $\begin{array}{r}1.31 \\
-1.01\end{array}$ & & \\
\hline $3 c$ & & & & .72 & -.71 & -1.01 & .621 & \\
\hline 30 & & & & & - & & .531 & \\
\hline 31 & & & & 6. & & -1.01 & & \\
\hline 317 & 1 & & & 6. & -.71 .50 & -1.01 & & \\
\hline 322 & 1 & & & 1.83 & -.411 .40 & $.6 !$ & .351 & \\
\hline $\begin{array}{l}32 \\
61\end{array}$ & & & & .72 & $-.71 \quad .50$ & -1.01 & .621 & \\
\hline & & & & : & $\begin{array}{r}-.21 \\
.01\end{array}$ & $\begin{array}{l}-.71 \\
-.11\end{array}$ & $\begin{array}{l}.451 \\
.291\end{array}$ & \\
\hline 302 & & & & .76 & -.61 & & .631 & \\
\hline 75 & 13 & & & .65 & -1.01 & -1.11 & & \\
\hline & & & & 6. & & & .151 & \\
\hline 31 & 16 & & & & & 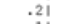 & & \\
\hline $\begin{array}{l}10 \\
10\end{array}$ & 16 & & & & & & & \\
\hline & 16 & & & & & & .281 & \\
\hline
\end{tabular}

Figura 6.

Tabla de valoración en unidades continuas para los usuarios 


\section{Conclusiones}

Con los resultados obtenidos a través de los ensayos realizados con las instancias de prueba, se pudo establecer que el modelo de elección discreta ejecuta de forma eficiente una adecuada discriminación del impacto que, en términos de la calidad de vida, tienen las cirugías programadas para los usuarios en lista de espera.

Con base en el índice calculado para cada usuario, el modelo pudo generar iteraciones de grupos óptimos de pacientes para el uso de las salas, acorde con los requerimientos de la IPS, estableciendo puntos de corte en la lista priorizada conforme a la capacidad instalada.

Los resultados de las simulaciones del modelo muestran consistencia en las valoraciones de los resultados individuales del nivel de calidad de atención para los pacientes, pues no se alteran por causa del entorno. Esto demuestra que el modelo es independiente de la población y del instrumento de medida.

El diseño del grupo de ítems que se usan como reactivos es un reflejo de la capacidad operativa de la institución prestadora de servicios de salud, y juega un papel crucial en el establecimiento de las prioridades.

Habida cuenta de lo anterior, se aporta un modelo de medición para que las personas que tienen a cargo la organización y priorización de usuarios en listas de espera, dispongan de un método que les ayudará a establecer las prioridades de atención para los grupos de usuarios que componen las listas de espera. Esta lista de prioridades asistenciales queda habilitada mediante una variable y una escala para que pueda ser evaluada frente a listas de viabilidad financiera en modelos multiobjetivo. A través de los ensayos realizados con diversos grupos de ítems se evidencia la vulnerabilidad del modelo ante una inadecuada construcción de la escala compuesta por los ítems, lo que hace que el diseño del instrumento se convierta en un factor de sesgo de mucha relevancia.

\section{Referencias}

Alemi, F. \& Gustafson, D. ( 2010). Decision Analysis for Healthcare Managers. Chicago : Health Administration Press.

Anderson, D. \& Sweeney, D. (2009). Estadística para Administración y Economía. México: ECengage Learning.

Apella, I. (2009). Estimación de la función de demanda de salud. El caso Argentino. Nuevos documentos CEDESD., 59, 16-19.
Asua, J. \& Taboada, J (2005). Experiencias e instrumentos de priorización. Red de investigación de resultados en salud y servicios sanitarios (IRYSS), 2-3.

Bond, T. \& Fox, C. (2001). Applying the Rasch Model : Fundamental Measurement in the Human Sciences. New Jersey: Lawrence Erlbaum Associates, Inc.

Briones, G. (2008). Métodos y Técnicas de investigación para las ciencias sociales. México: Trillas.

Cardoen, B. (2010). Operating room planning and scheduling: A literatura review. European Journal of Operational research 201(3), 921-932.

Cohen, L. (1979). Approximate Expressions for Parameter Estimates in the Rasch Model. The British Journal of Mathematical and Statistical Psychology, 32, 113-120.

Corporacion IBM (2016, 03 25). IBM Software SPSS. Retrieved from IBM Software SPSS: Recuperado de http:// www-01.ibm.com/software/co/analytics/spss/

De la Garza, J., Morales, B. \& González, B. (2013). Análisis estadístico Multivariante. México: Mc Graw Hill.

Derret, S., Devlin, N. (2003). Prioritizing patients for elective surgery: a prospective study of clinical priority assessment criteria in New Zeland. Int J of Technology Assess Health Care, 91-105.

Edwards, R. (1997). NHS Waiting Lists: Towards the elusive solution. London: Office of Healths Economics .

Espallargues, M., Gallo, P. \& Sampietro, L. (2000). Situación y abordaje de las listas de espera en Europa. Agència d'Avaluacióde Tecnologia i Recerca Mèdiques, Departament de Sanitat i Seguretat Social. Barcelona: Generalitat de Catalunya.

Habermann, S. (1977). Maximum Likelihood estimates in exponencial response models. The annals of statistics, 5 ( 8), 15-841.

Hair, A., \& Tatham, B. (2001). Análisis multivariante (5a ed México: Prentice Hall.

Hauch, K. \& Smith, P. (2004). The economics of priority setting for health care: a literatura review. Washington, DC (US): The world Bank.

Kenneth, E. (2009). Discrete Choice Methods with Simulation (2.a ed.). Cambridge: Cambridge. 
Kerlinger, F. \& Lee, H. (2002). Investigación del Comportamiento (4.a ed.). México: McGraw Hill.

Liao, T. (1994). Interpreting Probability models Logit, probit and other generalized linear models. Illinois: SAGE publications, Series on Quantitative Applications in the social sciences.

Linacre \& Wright, (s.f.). A User's Guide to BIGSTEPS Rasch Model Computer Program Recuperado de://www.winsteps.com/a/bigsteps.pdf

Ministerio de la Protección Social. (2008). Precios y Contratos en salud, Estudio indicativo de precios y análisis cualitativo de contratos. Bogotá: Colección PARS.

Ministerio de Salud. (2015, 06, 01ListadodelndicadoresdelPS.aspx http://calidadensalud.minsalud.gov.co/IndicadoresdeCalidad/Listadodelndicadores/

Montgomery, R. (1995). Probabilidad y Estadística Aplicadas a la Ingeniería. México: Limusa.

Ortún, V., Pinto, J., \& Puig-Junoy J. (2001). El establecimiento de prioridades. Atención Primaria, 673-6.

Oudhoff, J., \& Rimmermans, D. (2007). Waiting for elective general surgery:Impact on health related quality of life and psychosocial consequences. BMC Public Health, 164-174.

Pride, W. (2015). Foundations of Marketing. Stanford: Cengage learning.

Rasch, G. (1981). Probabilistic models for some Intelligence and attainment Test. Chicago: University of Chicago.
Sabik, L., \& Lie, R. (2008). Priority setting in health care: Lessons from the experiences of eight countries. Equity Health, 1-13.

Sallenave, J. (1994). La gerencia integral. Bogotá: Norma.

Sánchez, F. \& Abellán, J. (2008). Cómo se deben establecer y evaluar las prioridades en salud y en servicios de salud? Métodos de priorización y disparidades regionales. Informe SESPAS, 22 (Supl 1), 126-36.

Taha, H. (2008). Investigación de Operaciones. México: Pearson.

Thorndike, R. (1989). Psicometría Aplicada. p. 127. México: Limusa.

Tristán, A. (2001). Análisis de Rasch para todos México: Ed Ceneval.

Wright, B. (1977). Solving measurement problems with the Rasch model. Journal of education nmeasurement, $14,97-116$.

Wright, B. \& Douglas, G. (1976). A Rasch ítems analysis by hand. Research memorándum № 21 Statistical Laboratory. Department of education University of chicago, 1-6.

Wright, B. \& Mead, R. (1975). Sample-free calibration with a Rasch measurement model. Research memorandum $\mathrm{N}^{\circ}$ 18 Statistical Laboratory: Department of education University of chicago, 1-6. 